\title{
Preface: Two Perspectives
}

Decentralization increasingly affects business and society. Despite the work that led to this book and continues beyond these pages, the authors count themselves among those who continuously learn what decentralization can offer us. We believe that the journey into decentralization requires an iterative process that questions centralized approaches to otherwise unsolvable societal problems at every level. Beginning to unlearn a lifetime of centralization is the first step into an appreciation for the power of decentralization.

The authors care deeply about the prospect and potential of decentralized systems for the future of humanity. The passion for decentralized societal solutions is motivated by distinct yet supplemental perspectives on decentralization, which have inspired this book.

As an economist and lawyer, Wulf Kaal's scholarship has been motivated by a desire to create functional institutional designs since his early Ph.D. training days as a mentee of a leading scholar on new institutional economics. Over time, Wulf's scholarly interest in institutional design and governance morphed into work on the economic theory of dynamic governance, dynamic regulatory structures, crypto economics, decentralized infrastructure products, and incentive design mechanisms in decentralized systems, including via co-authored articles with Craig Calcaterra.

Craig Calcaterra is an expert on abstract dynamical systems in mathematics who brings a unique skillset to architecture and design mechanisms in decentralized systems. Craig's Ph.D. training in mathematics and particular expertise in control theory and stochastic dynamics gives him a useful perspective for modeling and analyzing global economic and social systems with their diverse moving parts and complex inner relationships.

The authors met when Craig's wife, Karen, decided to go back to school for a law degree and took a class that Wulf was teaching. In his course, Disruptive Innovation, Professor Kaal implored his students to build relationships with more technically proficient people, like computer scientists and mathematicians, because he was witnessing and anticipating the effect of new technologies on the legal profession. Karen thought, "I know a mathematician." After an introduction in which Wulf suggested the problem of using blockchain technology to create a decentralized arbitration platform, Distributed Jurisdiction, Craig decided that his midlife crisis might as well be a swan dive from the ivory tower of math academia into the muck of real life. Four years later we've decided to write a book detailing the insights we've gleaned tackling business and social problems by collaborating with our diverse perspectives.

The journey we've taken together is being taken every day, organically, by broader society. The clean and clear, logically coherent theory of hard science and mathematics needs to reconcile itself with the messy and confusing, logically inconsistent hard realities of international issues of economics and law and social science. The precise tools of computer science are being used and abused by humans, with all their diverse 
range of motivations - from idealistic generosity to malicious selfishness, from group scapegoating to individual aspiration - together on the same platforms, on social media, in international forums, and in cities around the world as diverse communities are merging in new social permutations.

In this book we hope to share some of the insights we've gleaned from computer science and graph theory, from game theory and category theory, from history and international relations, from social science and economics, applied to our chaotically churning technologically revolutionary moment in the present global business environment and social atmosphere.

\section{What is Decentralization? A Lawyer's Perspective from Wulf Kaal}

No two minds will agree on a common definition of decentralization. The word "decentralization" triggers different associations in different people, depending on the totality of their experiences, socialization, and socioeconomic upbringing. On the extreme ends, where one person will see opportunity, development, and a natural evolution, others will perceive risk, destruction, and societal doom.

Most people still see decentralization from the vantage point of centralized systems and hierarchical structures. That is very much understandable. Out of necessity, humans have engaged in centralized thinking since the emergence of tribal societies and throughout urbanization and the industrial revolution to today. Throughout history, people have been subject to the apparent chaos of natural decentralization and sought comfort in centralization. Centralization brings structure to the natural decentralized order of things. Centralization brings control, convenience, and efficiency.

Because of hierarchical structures, centrally organized entities allocate resources with minimal loss of energy and can identify and remove waste in the system. Centralized production methods efficiently reduce per unit costs. Centralized organization of society leads to efficient resource extraction, rent-seeking, and the creation of economies of scale. Yet, increasing evidence suggests that humanity's centralized economic expansion and associated natural resource extraction are unsustainable. Other indicators suggest that centralized organization of society has in many ways reached limitations: the general weakening legitimacy of the public sector; increasing economic decline; disillusionment with existing business, political, and social institutions; inadequate response to emergent geopolitical problems; and global and international pressure on countries with inefficient, undemocratic, overly centralized systems. Even centralized technologies that previously enabled more sustainable solutions encounter capacity issues. For example, the increasing connectivity of society via the internet has resulted, according to some estimates, in the need for decentralized authentication protocols. 
Decentralization is saving the world. Decentralized technology and ecosystems are correcting human-created centralized destruction. Humanity's unsustainable centralized economic expansion and associated natural resource extraction are counteracted by decentralized technology solutions. For example, the disillusionment with existing political institutions' ability to address economic hardships that emerged in the aftermath of the financial crisis of 2008-2009 gave rise to a new form of technological decentralization. The Bitcoin protocol emerged in 2009 as an attempt by its founders to provide a decentralized alternative to the shortcomings of the financial system.

Bitcoin and its progeny spawned a slew of additional decentralized protocols and decentralized technology attempts and solutions that provide a gateway to future forms of technological decentralization. The evolution of decentralized protocols, ecosystems, and platforms, in turn, may provide unprecedented democratic forms of organizing business and society and coordinating human behavior. The emergence and proliferation of distributed applications (DApps) in the aftermath of the invention of the Bitcoin protocol in 2009 demonstrate that a nascent market for such applications and consumer demand already exists. Consumer preferences will continue to shape the DApps market and the solutions it may offer for commerce and society.

Unlike other societal phenomena, decentralization appears as a natural default solution for societal issues. Change in business and society is often accompanied by drastic measures and long public debates along political lines. For example, the occurrence of globalization in technology, business, politics, and society, was subject to long drawn-out public debates about the possible effects and risks. By contrast, the decentralization of business and society is mostly a quiet occurrence without much media attention that appears as a default solution based on the existing centralized network infrastructure. Decentralization is incremental and iterative and a natural default solution that is based on existing networks. Existing centralized businesses and business networks are increasingly combined with decentralized elements.

\section{Definition?}

An understanding of decentralization depends to some degree on its delineation from its centralized counterparts. In other words, a definition of what centralization in a given context, industry, or field of study means also helps define what decentralization in this context can mean. The definition of decentralization changes based on the kind of decentralization, the industry or field, application, and the overall context. What may apply in the context of political decentralization may not at all be relevant in the context of technological decentralization. Organizational decentralization, market decentralization, societal decentralization, among others, all emphasize different aspects of decentralization. Yet, each different kind of decentralization may have knock-on effects on the others. Accordingly, a definition of decentralization necessitates an inclusive scope that derives from its core characteristics. 
Defining decentralization through historically formed centralized perspectives is a contradiction in terms. It undermines the true potential of decentralization. While even fully networked and decentralized systems are still subject to centralized elements, such as the agreement on certain terms and meanings in language, using a centralized understanding of a subject to explain what decentralization of that subject could mean limits the scope and scale of decentralized approaches. For example, decentralization is not just the addition of hierarchical levels in a centralized organization; decentralization is not just the redistribution of centrally organized authority or redistribution of centrally collected revenue; decentralization is not just the delegation of centralized authority to managers on all levels of an organization. Terminologically, decentralization is not just synonymous with delegation, deconcentration, disassortative, devolution, circulation, or partnership.

Even the basic societal norms instantiated in the subsidiarity principle cannot fully encapsulate the essence of emerging decentralization. The subsidiarity principle is a general principle of social organization. It suggests that social and political issues should be addressed at the most immediate level that is consistent with the resolution of the issue. Decisions should be made by the government entity that is closest to the populations affected by a given issue. The subsidiarity principle is deeply rooted in existing legal frameworks and it is a general principle of the European Union. While local government may be closer to the concerns of the people and better able to respond to the preferences of its citizens, subsidiarity alone cannot encapsulate the ontology and desirable outcomes of decentralization.

\section{Technical Delineation}

The degree of decentralization can be delineated by distinguishing several core concepts such as logical decentralization, architectural decentralization, and political decentralization. While these core concepts are distinguishable, some overlap is unavoidable.

An example from the legal system helps illustrate the overlapping concepts of logical decentralization, architectural or hardware decentralization, and political or governance decentralization. Both civil and common law are logically centralized as all decision-making power goes back to what the respective legal bodies ordained. For the most part, common law is based on legal precedents created by a diverse body of individual judges. By contrast, civil law relies mostly on a centralized parliamentary or legislative decision-making body. Architectural decentralization is disparate in civil and common law as common law has more decentralized power in the individual courts and jurisdictions that create the precedent. Governance decentralization is low in both common and civil law, as only a limited number of decision-making authorities, courts, and parliament have the ability to make changes to the body of law that apply to all of the law's subjects. 
The firm - that is, a traditional corporation - provides another example to delineate logical decentralization, architectural decentralization, and governance decentralization. The firm is logically centralized as a division of the firm would typically break it apart and it would not be able to operate independently as two separate parts. This is traceable to the firm's hierarchical order that creates efficiencies and structure. The firm is also architecturally centralized as it typically is governed through one physically centralized headquarter or office. The governance of the firm is also typically centralized as only a select few can make changes that affect the totality of individuals involved with the firm.

\section{Natural Decentralization}

Nature's ecosystem provides an illustration of natural decentralization. The natural ecosystem is composed of the community of living and nonliving organisms whose interaction is facilitated by chemical, biological, and physical processes in sunlight, air, water, soil, plants, microorganisms, and animals. No central organism or system dictates when, where, and how the interactions materialize and how living and nonliving organisms proliferate and evolve.

The natural ecosystem epitomizes the characteristics of decentralized systems. The intelligent order in the natural ecosystem is distributed throughout the system without central coordination. Information on environmental factors that influence organisms' existence and development naturally, filter into the edges of the ecosystem in real time. Random genetic mutation of organisms is further enhanced through dynamic feedback effects on environmental factors that benefit a given mutation and accelerate its survivability in that environment. Mutated and adaptable organisms become more attack resistant in comparison with the original population. In turn, the ability to mutate via feedback effects for enhanced adaptability allows the adapted organism to proliferate more quickly than the original population.

Nature provides systems that create spontaneous order. Nature provides several prominent examples of spontaneous order and coordination of self-organizing behavior. Slime molds, flocks of birds, ant colonies, and schools of fish exhibit emergent and self-organizing behavior in which interactions and feedback effects between selfsufficient individuals create spontaneous order. Unrelated eukaryotic organisms, aka slime mold, are capable of existing without constraints as single cells, but can also congregate to form multicellular reproductive structures. Spontaneous order is less likely to emerge unconstrained from interactions dispersed across a system if the system has a power or information center. Distributed self-organizing systems are nimbler and able to efficiently self-repair at points of local failure through coordination without control.

The natural ecosystem evolved randomly with an inherent order and interdependent structure. Take, for example, how crops grow in nature. Crops grow best 
where the conditions made available by nature are most ideal. Flora and fauna quasi-randomly interact with each other to effectuate that growth. Flora, that is, plant life, develops in a particular region or time; the corresponding fauna, that is, animal life, supplements that development and grows. Birds take seeds and crops from one meadow to another area. Or, the wind blows certain seeds across vast areas of land until they find more fertile soil, and so forth.

Interoperability and interdependence characterize the development of the natural ecosystem. Flora and fauna are interdependent. Animals cannot develop in a given part of the planet if they do not have access to the right kinds of food that enable their evolution and survivability. In turn, animals enable plants to flourish in parts of the planet where they previously could not exist.

The equilibrium in the natural ecosystem is facilitated by the decentralized coordination of interdependent organisms. The equilibrium in the natural ecosystem is not created by a centralized dictator who examines the needs of nonliving and living organisms. Rather, nature's equilibrium materializes through a balancing of the interactions in chemical, biological, and physical processes. Ecological systems are usually in homeostatis, that is, in a stable equilibrium. If a given part of the ecosystem gets out of balance, the system typically naturally self-corrects. The system corrects small changes, such as the size of a particular population growing too quickly, through negative feedback. The feedback brings the changed parameter in the system back to its original equilibrium. This facilitates that the changed parameter can again correspond optimally with the rest of the system.

The process of mitosis, or cell division, provides a natural precedent and overview of the role of governance in decentralized systems. Mitosis is the process in nature whereby the nucleus of a single cell divides into two identical daughter cells, which thereafter contain the same genetic information and functionality as the original cell. After mitosis, both cells are independently fully functional. Because of apoptosis, that is programmed cell death, cell replacement via mitosis is vital. The purpose of mitosis is growth, cell regeneration, and the removal of worn out or damaged cells. Without mitosis, organisms could not be functional and would die. The cell nucleus, that is, typically a single rounded structure bounded by a double membrane, containing the genetic material, enables the process of mitosis. Similarly, in decentralized systems, information, and any functionality that depends on information, is continuously changing. As parts of system information are being outdated by the naturally changing environment, they need to be replaced to keep the entire system operational. Like mitosis, this decentralized governance process has to be autonomous and automated in the system. Decentralized governance has to be able to evolve and mutate and create new information precedent for the continuous efficient operation of the system. The nucleus of the decentralized system is the information (contained in the code) that constitutes the architecture and incentive design of the decentralized operating system. It facilitates the information exchange. 
The standard model of particle physics provides perhaps the strongest argument against natural decentralized evolution. While naturally incomplete, the standard model of particle physics is the most complete model science created to describe the observable physical cosmos. It suggests that the entire physical cosmos can be traced back to a single point. Science has no answers as to what came before. If combined with the theory of natural selection, which suggests that all life on earth could be traced back to a single cell organism, the standard model of particle physics would counteract decentralized evolutionary theories. Yet, single point origin does not counteract the multiplicity of design mechanism in the following evolution.

Nature evolved through natural selection: a naturally decentralized selection mechanism. No centralized authority determines which organisms can survive when and where. Rather, the interaction of the community of living and nonliving organisms by way of chemical, biological, and physical processes and the totality of environmental factors dictates which species survive and flourish. Because natural selection is a complex process involving multiple interconnected causes with adaptation to the natural environment at its core, natural selection involves a given population with distinguishable characteristics. Variability is heritable. Random genetic mutations increase variability. No centralized order determines in what environment certain characteristics are ideally suitable for enhanced survivability. Rather, the population with the traits that are most adaptable and heritable has a natural comparative advantage. The natural selection of the most adaptable and survivable population characteristics materializes without a centralized mechanism. Even characteristics that appeared at the edges of a given ecosystem may be most ideally suited for survivability.

\section{Technological Extension of the Decentralized Natural Order}

The natural order emerged through decentralized coordination. Humans centralized parts of the decentralized natural order to enable human proliferation. In turn, increasing evidence suggests that technology enables a decentralized extension of societal organization and human achievement. Emerging decentralized technology could transform the digital universe into a complex adaptive system of the kinds found in nature.

Human utilization of nature provides a prominent example of centralized order in decentralized systems. Throughout history, humanity has been subject to the apparent chaos of nature and sought comfort in centralized control over it. The creation of property rights over the natural order of things enabled centralized efficiency for human achievements. Centralized urbanization provided protection and efficiency from the chaos of nature. Humans centralized parts of the natural order to create economies of scale that would otherwise not be possible within the natural order. For example, to control where the most beneficial conditions allow crops to flourish and result in a significant harvest, farmers try to control the environment and soil that allow their crops 
to grow optimally. Creating the conditions to grow most of a certain type of crop in the shortest amount of time allows farmers to more effectively control the output of their efforts and create economies of scale that would not be possible within the natural order.

The evolving decentralization of business and society is a form of a decentralized extension of the existing societal organization and human achievement. As organizational centralization reaches natural limitations, decentralized organizational elements become more prevalent. For example, internet-based platform businesses try to make organizational hierarchies flatter to increase creativity and output and instill a culture of "best idea wins." The farthest extension of these ideals is instantiated in the decentralized autonomous organization (DAO), an organization that only exists in code. Similarly, as business reaches natural frontiers, it extends its reach via decentralized structures. Companies like eBay and Amazon provide examples of centralized companies that decentralized the customer experience. These companies use the structure, control, and associated profit optimization of centralized entities and combine it with the bottom-up approach of decentralization in the form of customer reviews.

Decentralized technology is part of a natural extension of the decentralized origin of human tool-making. For example, just as language became a tool for the optimization of human outputs, decentralization can help coordinate and optimize human efforts and outputs. This is exemplified by emerging decentralized technology's ability to remove the inefficiencies and costs associated with intermediation. For example, cost free value transfer via decentralized technologies removes the cost for migrant workers. In 2019 worldwide remittance has replaced foreign direct investment in total value across borders.

Decentralized evolution is an extension of naturally decentralized development. As decentralized solutions morph and proliferate, they follow existing natural network patterns and precedents. Technology-driven decentralized systems depend on an existing infrastructure. Just as predator populations could not evolve in the natural ecosystem without prey, decentralized technology systems cannot evolve without an existing decentralized infrastructure. Generations of increasingly networked solutions enable an evolution of decentralized systems.

\section{Basics of Decentralization}

Decentralized systems depend on network effects. Network effects occur when increasing numbers of participants or users in a given network improve the value of network access for the entirety of the users. An example that illustrates network effects for an existing network is the internet. When the internet had very few users it was of limited value. As its user base increased, the totality of users benefitted from an ever-increasing use and application of internet connectivity as products and services on the internet proliferated. Similarly, eBay, the internet-based auction site, 
proliferated as more users offered their products for auction. The more people offer and bid, the higher the potential prices of products on eBay and the more new sellers are incentivized to join because they see that the totality of users on eBay enables them to sell their products. However, a network's infrastructure can compromise its network effects and associated user benefits.

Intelligence is widely dispersed throughout decentralized systems. Because of the lack of traditional coordination hierarchies and the lack of central intelligence, the information and intelligence in decentralized systems is more dispersed throughout the system. Information and knowledge exchange naturally occur and filter in at the edges of decentralized systems. No guarantee exists in a decentralized system that the randomly generated information is accurate. That is the case on Amazon and on the internet in general where there is no real attempt to clean the data. These are exactly the type of shortcomings future decentralized infrastructure products, such as a decentralized verification engine, need to address.

Information production is localized and subject to feedback effects in decentralized systems. Relevant information occurs randomly at the edges of decentralized systems and its relevance is determined by an initial group of users. The information is then transferred to other peers who realize the relevance of a given piece of information for their own needs. As the application of the information changes, depending on which groups and subgroups of peers in the decentralized network use it, the changed relevance of the original piece of information is then transmitted back to the original information generator who evaluates it and either applies it or further amends it. This feedback constantly updates and optimizes the quality of information.

Feedback effects enable decentralized coordination. The feedback effects in decentralized systems not only update and optimize the quality of information. They also increase the relevancy of the applications of the information. As peers in decentralized systems analyze a given set of information they receive, they also examine the application of this information for their own needs. For example, contributors in Wikipedia are engaged in the pursuit of knowledge in a constant feedback loop with other contributors pertaining to a given entry on Wikipedia. Similarly, the use of computer code as a template for a smart contract enables individual creators to benefit from the input of others. The code originates in a smaller subgroup of a peer-to-peer (P2P) network. The subgroup users examine the applicability of a given computer code template for their own uses. If the code passes muster, it may be included in a slightly changed or enhanced instantiation. The enhancement, in turn, may provide feedback to the originator group, and so forth. The feedback enables coordination of most relevant information.

The values and core belief systems of members provide cohesion and longevity in decentralized systems. For example, contributors to Wikipedia share a common belief in the power of the crowd, that decentralized collaboration for the pursuit of knowledge creates superior outputs. They collaborate because their values and core beliefs in knowledge creation unite them in a common cause. 
Independent and autonomous subgroups create the backbone of decentralized structures. Subgroups are formed randomly based on individual members' ability to contribute to the common cause of the group. Members of the subgroup are typically equal and trust one another. For example, Wikipedia members are making contributions to the common cause of knowledge development in a subgroup of individuals that contribute to a particular entry. Each individual has a certain expertise pertaining to the entry. Collaboration is independent and autonomous. Some members write articles, others edit, and still others further optimize the entry. Contributors to Wikipedia trust each other to create and edit entries for the pursuit of knowledge and each is motivated to contribute to the best of their abilities. Norms of behavior evolve organically from the community, supplementing the basic set of rules provided and enforced by Wikipedia to coordinate member conduct and create and reinforce trust in the subgroup.

As decentralized DAOs emerge through decentralized technology, the value systems in DAOs are a determining factor for membership and cohesion. The desire to be connected via a DAO to like-minded individuals has two value propositions. First, individuals elect to join a DAO and thus signal their desire to cooperate in a decentralized network. Second, individuals select their particular DAO based on their values and interests. DAO members will choose to join a DAO if it corresponds with their existing expertise, inclinations, knowledge base, and beliefs.

The apparent intelligence chaos increases attack resistance in decentralized systems. Centralized coordination enables attack coordination because the hierarchical structure allows the identification of a single point of failure. The apparent intelligence chaos in decentralized systems, where information filters in at the edges of the system in real time, allows it to morph constantly. Decentralized systems can very easily mutate because the information flow is optimized through dynamic decentralized feedback effects. That ability to change without central coordination increases the attack resistance of decentralized systems.

Decentralized systems are more attack resistant, allocate information more effectively and use feedback effects for superior learning effects that optimize performance over time. Community and the efficient sharing of resources are more important in decentralized systems.

Centralization and decentralization are foundationally different approaches. Where centralization attempts to make sense of and create order in presumptive chaos, decentralization thrives in perceived chaos. Where centralization creates efficiencies via order, inefficiencies open new opportunities for participants in decentralized networks. Where centralization orders information, and presses it into a certain form, decentralization takes information from random places and utilizes it in new applications. Where centralization limits creativity through preset processes, decentralization frees the creative process. Where centralization creates hierarchies and substructures, decentralization removes hierarchies and structures and reorganizes in new ways. 


\section{What is Decentralization? A Mathematician's Perspective from Craig Calcaterra}
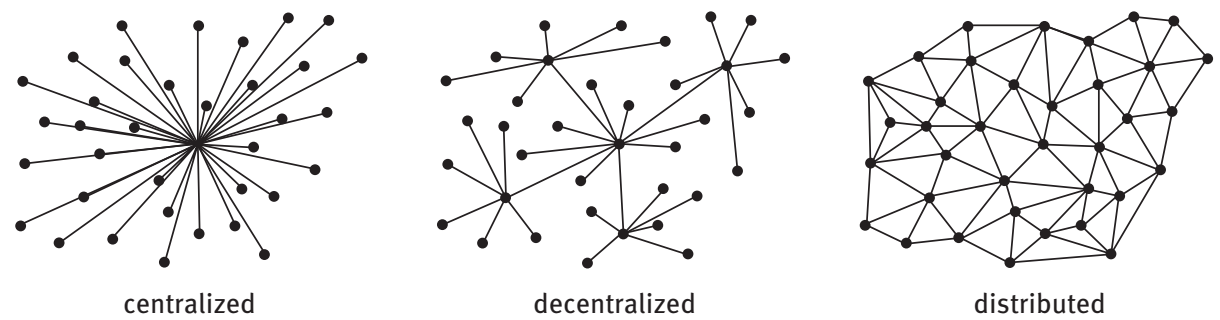

Figure 1: Common answer to the question, "What is decentralization?"

“The above image says it all.”-Rahul Behera, Content Director for Cryptosomniac. com. Top answer March 9, 2018, Quora

No, it doesn't.

Figure 1 may be a good first attempt at explaining decentralization. But it's vague and misleading. The closer you look, the more flawed it is. This figure is reproduced in many explanations of distributed computing, blockchain, or Web3. If you are going to learn anything from this book, at the least let it be how this figure is a shallow answer, and where it is wrong. ${ }^{1}$

Centralized means there is one person who is ultimately responsible for all decision-making - "The buck stops here." Or there is one computer in charge of how information is processed. The first graph in Figure 2 indicates how all the dots (which represent nodes, or members of the network) must answer to the central node. $^{2}$

1 The word distributed is often conflated with decentralized, for good reason, because distributed means IT duties (information storage or processing or communication) are spread across many different nodes. But the concepts of distributed and decentralized shouldn't be contrasted. Decentralized networks are always distributed; distributed networks may or may not be under centralized control. So "distributed" doesn't even belong in the same image.

2 A note about nodes: Put simply, nodes are the little dots in any graph. Nodes represent the members of the network. Computer scientists often think of nodes as the different computers connected in a network. The number, variety, and connectivity of the nodes in a system impact how data is generated, the processing power needed, and the diversity of data produced. The variety and connectivity determine how much information can come from a network. Decentralization means redundantly and flatly connected. The more connections a network has, the more resilient it is, and information exchange increases. 
Centralized

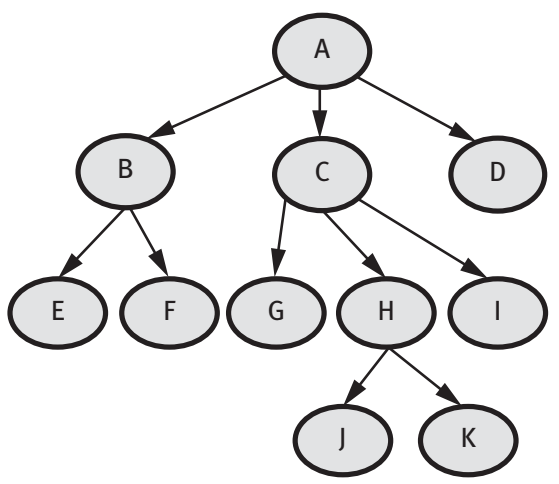

Decentralized

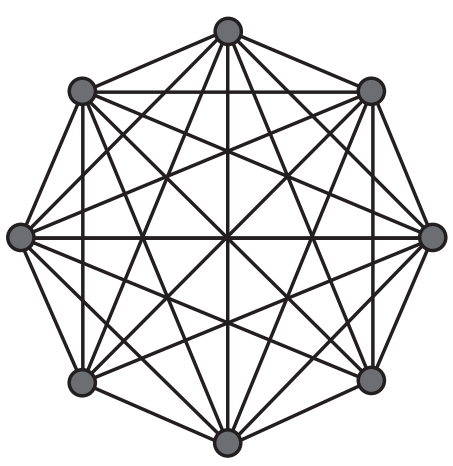

Figure 2: A better answer to the difference between centralized and decentralized. The ideal centralized configuration is a directed tree/hierarchy. The ideal decentralized configuration is a complete, undirected graph.

The ultimate centralized network is a tree graph, for example, an org chart in a company. It has a root to the tree, the leader. And it has branches that take orders from the root.

This left image of centralization in Figure 3 might represent the power structure of a god, which may be a good description of the experience of a typical app when the user is at the owner's mercy.
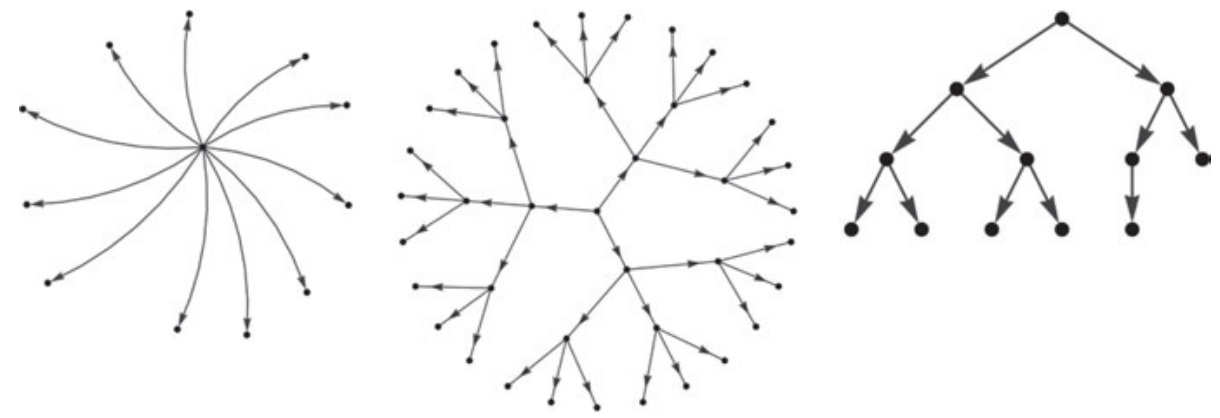

Figure 3: Diverse structures can display centralized organization, but they always exhibit an ultimate position of authority, the leader or root of the tree.

An org chart or a tree graph representation of a centralized organization plainly displays the advantage of a hierarchical structure. Everyone has a clear role in the organization. Their responsibilities and power are obvious from their position. The carrots and sticks can be clearly arranged, efficiently. In fact, no topology is more 
efficient for this purpose. ${ }^{3}$ Decisions made by a central leader can be carried out quickly and effectively.

Therefore, the best mathematical representation of a centralized hierarchy is a directed tree graph. The power flows only one way. In Figure 4, power is represented by the arrows flowing from top to bottom.

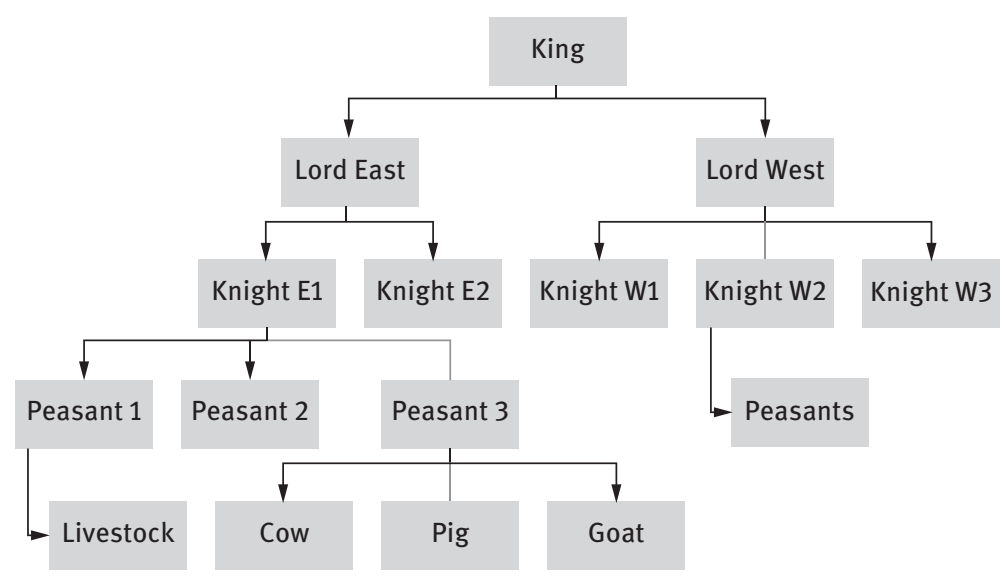

Figure 4: Feudal control centralization; part of the Great Chain of Being.

Hierarchical centralization is optimally efficient for making big decisions that affect the entire organization, and so it has historically outcompeted every other organizational structure, be it corporation or army or government. A hierarchy seems to eventually always emerge, or else the group fails.

Except that's not entirely true. Centralization comes with two major flaws that inevitably lead to its downfall.

First, every centralized organization has a single point of failure. If the leader is lacking in information, or skill, or wisdom, or charisma, the organization is bound to their poor decisions. ${ }^{4}$ In this age of voluminous and immediate information, business and social circumstances change much faster than ever before. An organization cannot wait for its leader to make a decision on every new choice the group faces. What is the alternative?

Secondly, the very success and efficiency of a hierarchy leads its members to become dependent on it, relying unquestioningly on the institution's design. The

3 Topology is a fancy word for structure, or the pattern of the arrangement of a system. The mathematical meaning is difficult to pin down without more technical definitions, but it roughly means the pattern that underlies a system when you ignore irrelevant details.

4 This is a major reason monarchies have fallen within a few generations of foundation throughout history. 
reliable answer to every question is "chain of command." People eventually place so much faith in the structure, it ossifies and becomes rigid. Then, while its structure may still be capable of handling some old, predictable tasks that the chain of command was originally designed to solve, the hierarchy may become too inflexible to reorganize and respond effectively to a novel crisis. (History is the record of the collapse of hierarchies. Examples include the Catholic Church in response to the Protestant Reformation and the Qing dynasty in response to Western imperialism.) What is the alternative?

Decentralized organizations thrive in both circumstances when choices multiply without bound and when novel crises arise.

Decentralized simply means "not centralized," so there is no dominant node, no leader. An example is the architecture of the internet, which was designed so that control is extremely redundant. If any local cluster goes down, the network will be able to continue functioning without a hiccup. No part of the network is crucial. No part of the network is the absolute authority. There is no president of the internet.

The notion of decentralization is captured by any graph that does not have a single node from which all power flows. The extreme form of decentralization is illustrated with a completely connected graph as in Figure 5. The goal of most decentralized networks is to achieve such interconnectivity and flatness of power distribution or complete equality of its members.
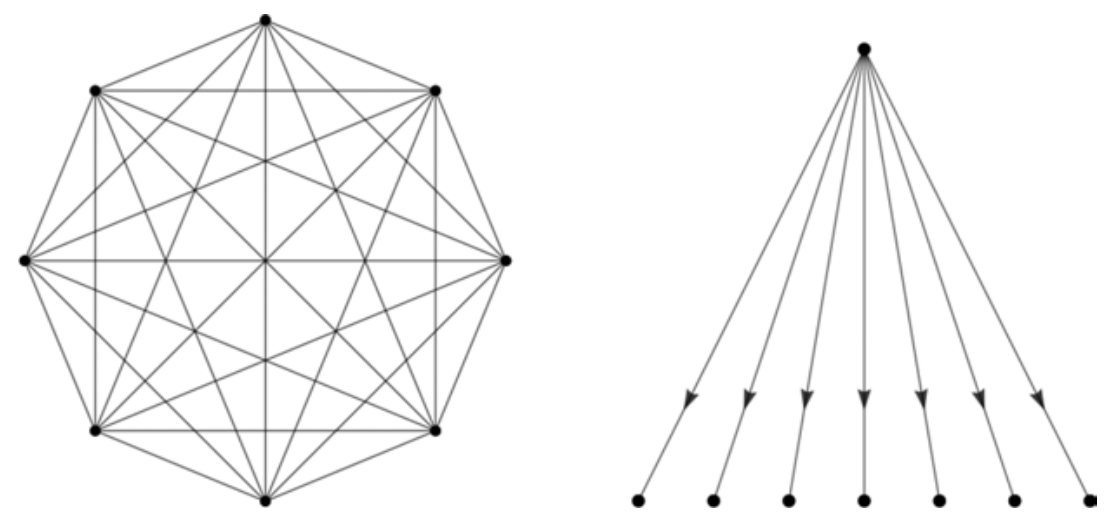

Figure 5: A complete graph with eight nodes versus simple tree with the same number of nodes but far fewer connections - stability versus efficiency.

\section{The Spectrum between Control and Freedom}

There is no clear-cut, discrete difference between centralized and decentralized. There is a spectrum between the two that any organization will fall along. The essential differentiator is the freedom of each member in the network. 
In the extreme of centralization, nodes/members have no autonomy, no freedom. Each node has a clearly defined role in the hierarchy, with explicit instructions for every behavior, in how and when they can act. With recent advances in information technology, we can now build dystopias of extreme centralization to a degree that makes George Orwell's society from 1984 seem trivial.

In the extreme of decentralization, every member has complete autonomy and freedom. Each member has complete independence, choosing how and whether to participate at any given time. With the recent advances in technology, we can now build systems that give members autonomy approaching this extreme, which still allows cooperation and harmony.

The most successful organizations choose a hybrid of centralization and decentralization, which maximizes the strengths of each.

\section{Dimensions of Decentralization}

Networks can be decentralized along many independent dimensions. ${ }^{5}$ For example:

\section{Location decentralization}

For example, Wikipedia's network of editors who may occupy any geographical location in any country with an internet connection. At the other extreme a classical corporation might require all employees work in the same building (see Figure 6).

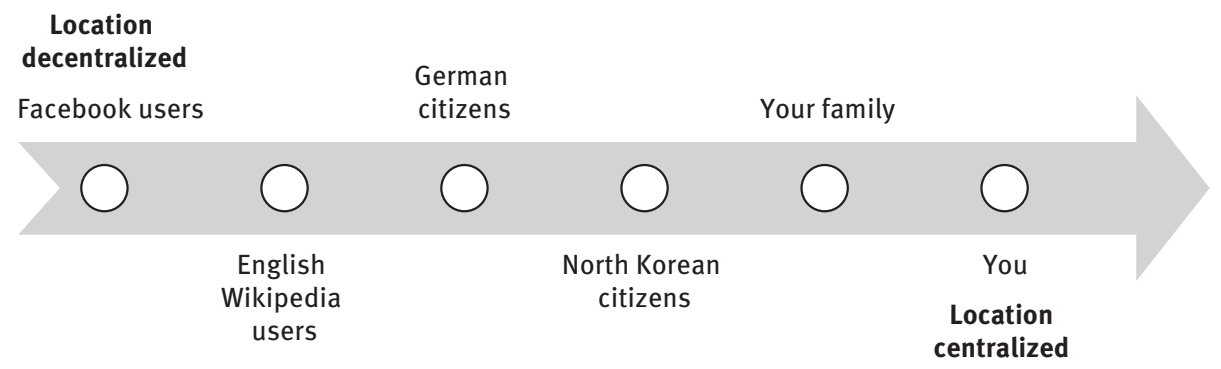

Figure 6: Spectrum of location decentralization.

\section{Control decentralization}

For example, political power, computational power, wealth, fame, etc. As of 2020, Wikipedia still has an ultimate arbiter in its founder, Jimmy Wales, and so it is not

5 Cf., Vitalik Buterin, “The Meaning of Decentralization,” Medium.com, February 6, 2017. https:// medium.com/@VitalikButerin/the-meaning-of-decentralization-a0c92b76a274 (retrieved 5/10/2020). 
completely decentralized politically. The English language, on the other hand, evolves without any ultimate authority on what new words can be added. Theoretically, anyone can invent a new English word, so it is completely politically decentralized (see Figure 7).

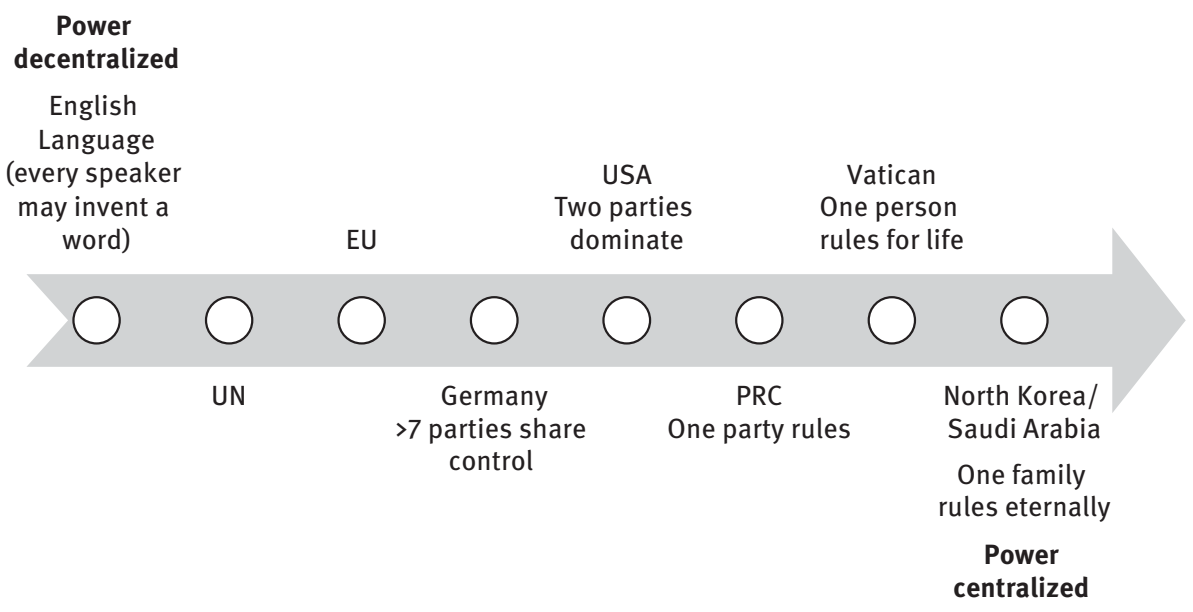

Figure 7: Spectrum of power decentralization.

\section{Protocol decentralization}

Is there a set of rules that everyone in the network submits to? Do all members follow the same logic and believe the same history? Almost every democracy in the West follows a set of laws more clearly specified than that of supposedly autocratic China, which has no formal constitution; its regular Five Year Plans are actually abstract aspirations for the country that each province interprets as best they can. Alternately, the French language has more rigid rules than English, so French is more protocol centralized than English. On the other hand, the French legal system is less formal than the British - French judges are less restricted by precedent and statute. Black market organizations can fall anywhere on the spectrum, but typically have much less formality in their rules than the English language does. Generally, at least some level of protocol centralization is required for group harmony and efficiency. We think of power decentralization as independent of protocol decentralization, but power determines how protocol is amended and enforced (see Figure 8).

We can further characterize most any quality along a (de)centralization spectrum. Centralized just means concentrated; decentralized means spread out. Events can be centralized or decentralized in time. Colors can be spread out along the light spectrum: a rainbow is color decentralized; a laser is color centralized. The ideals of a community can be focused or vague. 


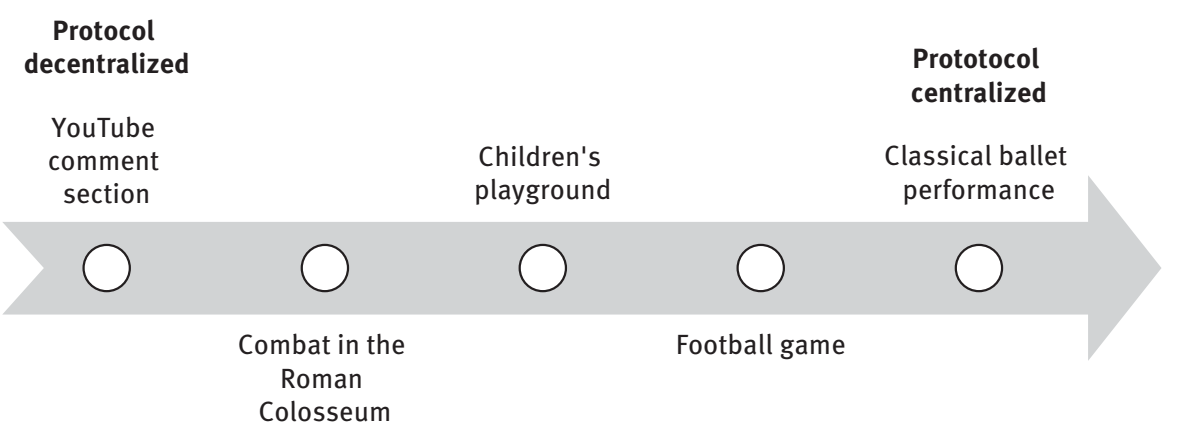

Figure 8: Spectrum of protocol decentralization.

\section{Spiritual ideals decentralization}

Peoples' beliefs may be more strictly focused or loose. For example, the Jewish religious belief spectrum ranges from ideologically rigid (centralized) to loose (decentralized) as illustrated in Figure 9.

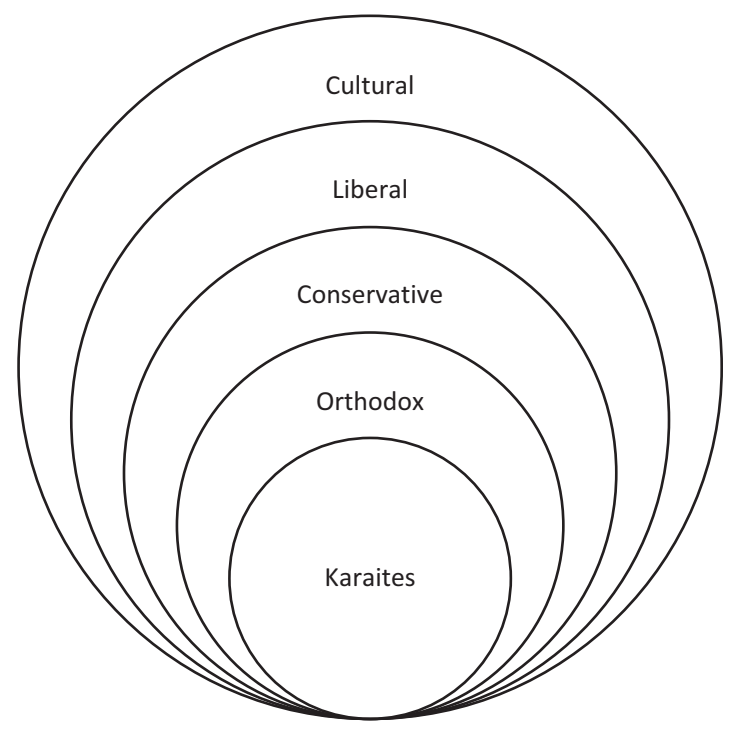

Figure 9: Spectrum of Judaic ideological adherence.

This oversimplified characterization presents the categories from more decentralized to more centralized as:

Cultural Jews - Identify with the culture and history of Jewish people, but not required to believe in any traditional religious tenet, or follow any specific strictures. 
Reform/Liberal Jews - Go to synagogue occasionally; believe in the general outline of the religious tenets but allow for alternate interpretations.

Conservative Jews - “Torah was inspired by G.d.” Laws strictly observed.

Orthodox Jews - "Torah was written by G.d.”

Karaite Jews - Respect only the written Torah with strict fundamentalist interpretation. Reject commentary such as Midrash or Talmud.

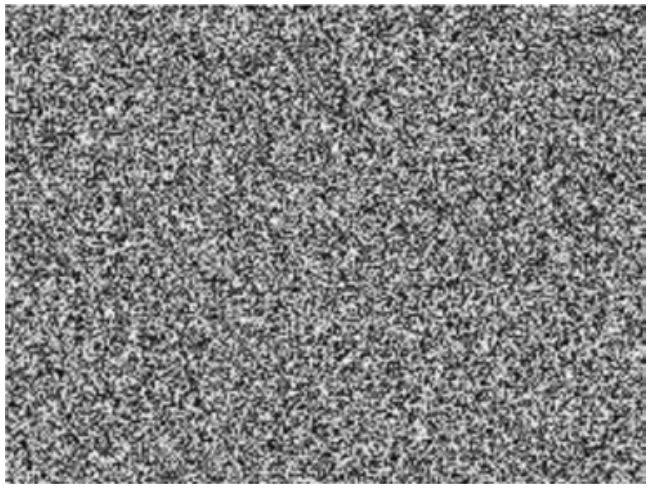

Figure 10: Extreme location decentralization.
Figure 11: Extreme location centralization.

Decentralization means spread out (see Figure 10).

Abstractly, centralized means concentrated (see Figure 11).

\section{Qualities of Decentralization: Stability, Efficiency, and Versatility}

From the abstract perspective illustrated in Figure 10 and 11 we see the fundamental qualities that determine the importance of centralization versus decentralization in any application.

Decentralization is stable. Centralization is unstable. A highly concentrated object, or a structure with a great deal of complicated order, has much more potential to be destabilized. Little changes can result in major structural change. On the other hand, if you disturb something that is extremely spread out, it's still spread out. The more concentrated a structure is, the more unstable it is. The more spread out a pattern is, the more stable it is - like a stool with one central leg versus the more stable arrangement of many legs spread out.

Decentralization is versatile. Centralization is task optimized. Given a specific task, centralized organizations are more effective at the task they are designed for than decentralized organizations. Getting a centralized group to move in a single direction 
takes much less effort. An optimally centralized structure would have no redundancy. So centralized organizations are more efficient at solving their singular problem. Decentralization is less effective; it generally takes longer to find the right components within its network, which can solve the problem. The more decentralized an organization, the more redundant it usually is. A decentralized organization is less efficient at solving a singular task. Communicating with and convincing each autonomous member of a decentralized group is very energetically intensive - "herding cats."

However, decentralized organizations are more versatile. If you ask the BMW Motorrad company to make a new type of motorcycle, they could obviously complete the job much more efficiently and effectively than the Wikipedia community. But if you want to find a cure for a disease, invent a new type of fusion cuisine, or host a diplomatic summit between African nations, then which group would fare better? Neither Motorrad nor Wikipedia are designed to solve those problems. But the centralized Motorrad organization would waste a lot more resources and probably produce inferior results to Wikipedia's. Wikipedia could tap the potential of its diverse members, harnessing the information at the edge, to identify the appropriate talent to solve a novel problem. It is reasonable to assume BMW's members are not less talented than the average Wikipedia contributor. But the BMW hierarchy would be an obstacle to recognizing which of the many ideas the group might propose to solve the novel problem best. The hierarchy would prevent the identification of talented people if they occur at the lower rungs. And it would resist any changes needed to construct a new hierarchy for organizing the effort. Since Tesla Motors is facing dynamic challenges with inventing new technology and production processes, they have consciously chosen to decentralize many aspects of their organization in order to maximize their versatility, so they can respond rapidly and efficiently to innovations and disruptions.

From a wider perspective, decentralization is efficient; centralization is inefficient. In any market, centralization is dangerous. Monopolies ruin the efficiency of a market - they impair its liquidity. The most efficient markets, the most liquid markets, have high transaction rates of many goods moving between many small players. Decentralization makes truth discovery more accurate, more reliable, and more efficient. Think of the difference between 10 competing news media companies reporting on a story versus 1 news company reporting 10 times. Or a physics experiment where a single researcher measures the speed of light 10 times with the same instrument versus 10 different researchers using 10 different instruments. Averaging the results is much more accurate when there are diverse contributors. This spawns the phrase, "the wisdom of the crowd." Decentralization is both stable and efficient. Ever since the writings of 18th century Scottish philosopher Adam Smith, it has been recognized that decentralization makes the wider economy more efficient.

The goal of every organization is to be effective, efficient, and versatile. Organizations select different dimensions around which they are centralized or decentralized. Corporations tend to have centralized power, because they wish to employ a 
singular strategy to efficiently exploit the circumstances of the market. However, this leads to instability whenever the market swings. A brittle hierarchy may crash before it can reorganize to effectively handle a new challenge. Decentralized organizations don't suffer swings in the market. A sufficiently decentralized organization is the market.

\section{Why and How?}

When is decentralization preferable to centralization? Why have we recently been hearing about decentralization more and more? What has changed technologically to allow decentralization to outcompete centralization? How can we decentralize our institutions, and why would we wish to?

In this book we will discuss centralized and decentralized organization in the past, present, and future. We will see how these notions have affected society throughout history, using the examples of Ancient Egypt and China to see how stability was maintained with protocol decentralization in the justice system. We will see how the Apaches used political decentralization to withstand successive empires devoted to their downfall. We will explore how a decentralized group of Jewish traders built a business network that thrived thousands of miles along the dangerous and chaotic Silk Road a thousand years ago with rudimentary information technology tools. We will analyze how 18th century Western democratic governmental designs united nations of diverse peoples into decentralized organizations. Using these historical examples, we can understand success and failure of organizations in the present, and how these ideas can predict the course of business and culture in the future.

In particular, we will explore the power of decentralization unleashed by leaps in computational power, information storage, internet communication, and cryptographic security. Decentralized power was leveraged recently by centralized corporations like Facebook and Google and Alibaba, making them the most powerful companies in the world. P2P technologies such as blockchain and distributed hash tables are being used to decentralize power further. Smartphones are empowering individuals worldwide with equalizing computation and communication power. The open source programming movement is fostering a culture of transparency, which results in tangible technological progress through meaningful cooperation, fulfilling the promise of democracy, while increasing security. ${ }^{6}$ Using the power of decentralization, we can create institutions that will bring harmony and stability to the new global society that is emerging. Designed wisely, such institutions will protect individual rights and empower us to cooperate for the benefit of all.

6 Cf., Linus' law, “Given Enough Eyeballs, All Bugs Are Shallow,” named after Linus Torvalds, the inventor of the open source operating system, Linux, coined by Eric S. Raymond, The Cathedral and the Bazaar: Musings on Linux and Open Source by an Accidental Revolutionary, O’Reilly Media, 1999. 